\title{
Diagnosis and Management of Systemic Lupus Erythematosus: A Case Report
}

\author{
Mohammed Huneif
}

\begin{abstract}
Systemic lupus erythematosus (SLE) is a multisystem inflammatory disease with a broad clinical presentation, which is principally difficult to diagnose across the emergency departments (EDs). The immune system of the body in this disease mistakenly damages or attacks healthy tissues. Majority of the patients suffering from SLE tend to develop "secondary heart disease" once in a while throughout the course of their primary illness. This study aimed to report a case of a previously healthy 9-year-old Saudi female who presented with rheumatic fever and congestive heart failure accompanied by productive cough, chest, abdomen, and back pain. A 9-year-old Saudi female was presented to the emergency department with a history of progressive rheumatic fever, iron deficiency anemia, and pain in chest with productive cough. Examination revealed that patient felt extremely ill, pale, afebrile, with a loss of appetite, tachycardic, high grade fever $\left(39^{\circ} \mathrm{C}\right)$, tachypneic, and a peripheral oxygen saturation of $95 \%$ on $40 \%$ supplemented oxygen" with low blood pressure 105/70 was noted. The patient was assumed to be diagnosed with probable SLE. We started our patient on methylprednisolone, omeprazole, and prednisolon and noticed sustained improvements. Multisystemic and acute life-threatening conditions should arise the suspicion of autoimmune diseases, predominantly SLE in the ED. SLE treatment shall be planned separately with consideration to utilize the "best-suited therapy" for targeting the organ systems affected. Lack of an explicit biological marker, disease heterogeneity, as well as absence of a specific outcome measurement for improvement makes this procedure harder.
\end{abstract}

Keywords: Rheumatic fever; Juvenile rheumatoid arthritis; Lupus erythematosus; Purpura; Discoid; Thrombotic thrombocytopenic

\section{Introduction}

Systemic lupus erythematosus (SLE) is considered to be a "multisystem inflammatory disease" which is often hard to di-

Manuscript accepted for publication February 23, 2017

Department of Pediatrics, College of Medicine, Najran Univeristy, Kingdom of Saudi Arabia. Email: huneif@hotmail.com

doi: https://doi.org/10.14740/jmc2782w agnose [1]. Specifically for the emergency department (ED) staff, it is critical to consider SLE as an option, when they observe an individual suffering from the signs and symptoms suggesting a disorder involving multiple systems. The complications observed in the EDs for SLE can be managed in a usual manner, the most widely found being pulmonary emboli, respiratory distress, hemoptysis, acute myocardial infarctions, and strokes $[2,3]$. Various other complications including cerebritis, renal failure, pulmonary hemorrhage, and pericardial tamponade can be managed by proper consultations obtained from the subspecialist [1].

Congestive heart failure in patients with SLE is often multifactorial in origin [4]. Individuals having lupus possess a significantly high risk for stroke, atherosclerosis or premature "coronary heart disease" (CHD) and many other "cardiovascular-related conditions" as compared to those deprived of lupus [5]. During the onset SLE in childhood, there are numerous clinical symptoms more specifically found in comparison to adults, such as proteinuria, renal involvement, mucocutaneous involvement/ ulcers, malar rash, seizures, urinary cellular casts, fever, hemolytic anemia, thrombocytopenia, and lymphadenopathy. Among adults, sicca as well as Raynaud pleuritic are thought to be twice as common as in adolescents and children [6]. One such classic presentation of a rash, joint pain, and a triad of fever in females of childbearing age needs a rapid investigation into SLE diagnosis. People with SLE often experience a broad spectrum of symptoms as well as have multiple combinations of the organs involvement, while no definite test can establish the systemic lupus diagnosis [7].

The management along with the diagnosis of the congestive heart failure in SLE is not different significantly from the results obtained through other etiologies. For the purpose of reversing this procedure, prompt recognition by laboratory evaluation, signs and symptoms, with echocardiography, chest $\mathrm{X}$-rays, and electrocardiogram is essential. To assist health care professionals to improve the SLE diagnosis accuracy, 11 such criteria were identified by the American Rheumatism Association [8]. These include discoid skin rash (redness in patches with hypopigmentation and hyperpigmentation tends to cause scarring), arthritis (more than two tender and swollen joints of the extremities), malar (observed on face mainly over the cheeks) "butterfly" rash, irritation of brain (manifested by psychosis or seizures referred to as "lupus cerebritis"), pericarditis or pleuritis, mucous membrane ulcers, antinuclear antibody, blood-count abnormalities, photosensitivity (rash observed in skin in reaction to exposure to ultraviolet light or sunlight), 
Table 1. Diagnostic Criteria of SLE [9]

\begin{tabular}{ll}
\hline Criterion & Definition/examples \\
\hline Malar rash & $\begin{array}{l}\text { Fixed erythema over the malar eminences, } \\
\text { tending to spare the nasolabial folds }\end{array}$ \\
Discoid rash & Erythematosus raised patches, may scar \\
Photosensitivity & Skin rash as a result of unusual reaction to sunlight \\
Oral ulcers & Usually painless \\
Arthritis & Non-erosive: Jaccoud's arthropathy \\
Serositis & 1) Pleuritis - pleuritic pain, pleural rub, pleural effusion \\
Renal disorder & 2) Pericarditis - ECG changes, rub, pericardial effusion \\
\hline Neurological disorder & 1) Proteinuria (>3+ or 0.5 g/day) \\
2) Cellular casts in urine \\
Hematological disorder & 1) Seizures \\
2) Psychosis \\
\hline Immunological disorder & 1) Hemolytic anemia \\
\hline Anti-nuclear antibody & 3) Leukopenia \\
\hline
\end{tabular}

A person is said to have SLE if he/she meets any four of these 11 criteria simultaneously or in succession.

and abnormalities in kidney (abnormal volume of urine protein or cellular elements clumps called casts measureable with a standard urinalysis) (Table 1) [8,9]. This study aimed to report a case of a previously healthy 9-year-old Saudi female who presented with rheumatic fever and congestive heart failure accompanied by productive cough, chest, abdomen, and back pain.

\section{Case Report}

A 9-year-old Saudi female was admitted due to rheumatic fever (experienced at the age of 6 years) accompanied by iron deficiency anemia, juvenile rheumatoid arthritis (6 years and 7 months), and celiac disease (reported at 8 years of age). Two weeks prior to admission, she presented with fever along with productive cough, paleness, chest, abdomen, and back pain, loss of appetite, depressed mood, poor school performance, malar rash, and mouth ulcers.

On inspection, patient was feeling extremely ill, pale, afebrile, with a high grade fever documented to be $39^{\circ} \mathrm{C}$, tachypneic, tachycardic, and a "peripheral oxygen saturation of $95 \%$ on $40 \%$ supplemented oxygen" with low blood pressure (BP) $105 / 70$ was noted. The patient had a history of juvenile rheumatoid arthritis, also showed typical congestive heart failure as of accepted case from the peripheral hospital at the age of 6 years, iron deficiency anemia, celiac disease and 2 months history of "inflammatory polyarthralgias" (joints pain) encompassing initially "interphalangeal joints", evolving, sometime later, generalized, progressive intermittent abdominal pain and fever ( 1 month), colicky in nature with no aggravating or relieving factors. At the age of 8 years, patient perceived failure to thrive and had frequent abdominal pain. Therefore, celiac profile and endoscopy was performed confirming celiac disease. One month prior to admission, she had symptoms of an upper respiratory tract infection followed by right and left ankle joint pain experienced for 2 weeks, both knees and wrist joints experienced for 1 month as migratory joints pain. For 1 month before admission, she was immobile (unable to walk) with severe pain. Back pain for 4 days increased with movement.

Her vital signs on presentation were noted for tachycardia 105 beats per minute, temperature $39^{\circ} \mathrm{C}$, BP 105/70 mm Hg, no distress, and scaring from the skin rashes reported previously. Neurological and respiratory examinations were otherwise average with $\mathrm{RR} 26 / \mathrm{min}$, and $\mathrm{O}_{2}$ saturation $92 \%$.

Initial laboratory tests demonstrated anemia: hemoglobin of $7.5 \mathrm{~g} / \mathrm{dL}(12.0-16.0 \mathrm{~g} / \mathrm{dL})$, platelets $798 \times 10^{9} / \mathrm{mL}$, white blood cells (WBC) count of $12.9 \times 10^{3} / \mu \mathrm{L}$, total bilirubin of $0.7 \mathrm{mg} / \mathrm{dL}$, undetectable direct bilirubin, glucose of $120 \mathrm{mg} /$ $\mathrm{dL}$, and $\mathrm{pH}$ 7.40. Serology for human immunodeficiency virus (HIV), hepatitis, and direct coombs tests were also found to be negative. Based on these findings, renal biopsy was performed and diagnosis of SLE was made and therapy with lasix, captopril, antibiotics, nebulization hydroxychloroquine (plaquenil) $200 \mathrm{mg}$ TW, vitamin D3 800, and international units PO once daily was initiated. We also continued methylprednisolone 30 $\mathrm{mg} / \mathrm{kg} / \mathrm{dose}, 1 \mathrm{~g} / \mathrm{day}$, given over $60 \mathrm{~min}$, for 3 days, omeprazole $1 \mathrm{mg} / \mathrm{kg} /$ day, and also started prednisolon $10 \mathrm{mg}$ PO OD for 1 month, with tapering doses prior to discharge. 


\section{Discussion}

The patient's prognosis with the SLE or SLE is known to be considerably improved with 20 years of survival now roughly about $80 \%$ partly owing to the treatments which are effective [10]. SLE is thought to be a "multisystem autoimmune disease" that tends to impact different organs as well as tissue in an individual's body ultimately causing dysfunction and damage. Some of the patients with lupus often possess mild disease that can primarily be treated with simple medications, while others tend to have life-threatening and serious complications. Treatments should be extremely individualized as well as varies as per the inconsistency during the clinical presentation of a disease [11]. The utmost care for the patients with SLE comprises of support and education services in addition to the non-pharmacologic and pharmacologic treatments. For SLE, the drug therapies are formed to suppress the inflammation and immune responses.

Establishment of the patient management and diagnosis with SLE needs an incorporation of the results of physical examination, patient symptoms, and diagnostic test results. The SLE management is mainly based on the disease manifestation and severity [12], even though "hydroxychloroquine" plays a dominant role in all SLE patients considering its treatment in long term. The "lupus in minorities: nature versus nurture" or (LUMINA) study along with other trials offers evidences of a decline in prolonged life and flares in individuals given hydroxychloroquine, thus, creating it as a foundation for managing SLE. Specifically in the patient, the diagnostic criteria of SLE were accomplished when oral ulcers, malar rash, serositis renal disorder: lupus nephrites II hematologic disorder, microcytic hypochromic anemia, immunologic disorder, anti-DNA antibody positive, anti-Smith abs positive, antinuclear antibody positive were summed up.

The EULAR also is referred to as "European League against Rheumatism" released recommendations for SLE treatments in 2007 [13]. In individuals with SLE without the manifestation of the major organs, antimalarial and glucocorticoid agents are considerably useful. However, for short term, NSAIDs can be consumed in patients possessing a low risk for complications from such drugs. Considering different immunosuppressive agents such as methotrexate, mycophenolate mofetil, and azathioprine in refractory circumstances or at times when doses of steroid cannot be limited to levels for a longer-term practice [14]. The recommendations from EULAR for SLE management with neuropsychiatric manifestations support the treatment and evaluation of these symptoms in a similar manner, as they are treated and evaluated in patients without SLE; if such symptoms persist, management of these symptoms as an extension of SLE should be considered [13].

\section{Conclusion}

The current case report implicates that SLE should be considered during refractory heart failure to substantial conventional therapy, exclusively in young females. Furthermore, early cor- ticosteroids treatment, either with or without immunosuppressive agents, might lead to improved and good outcomes. SLE treatments need to be planned on individual basis with primary consideration for utilizing best suited therapy so as to target the organ systems affected. Lack of certain biological markers, heterogeneity of the disease as well as absence of single outcome measurement for improvement often makes it a critical process. Lastly, this case specifically reminds a healthcare professional that even though lupus is considered to be a complicated disease on its own, some number of the patients do present with a more concomitant or second autoimmune disease that increases the complication. Due to this reason, the lupus diagnosis is just the starting point of a case. Furthermore, there is a need to raise awareness among SLE patients regarding this condition in order to guarantee concordance with treatment, as limited compliance subsidizes towards failure of treatment with flares in disease, accumulation of impairment such as amplified risk of death or renal failure.

\section{Acknowledgments}

The author is grateful to the University of Najran for their irreplaceable support.

\section{Funding}

None.

\section{Competing Interest}

The author declared that they have no competing interests.

\section{Consent}

Written informed consent was acquired from the patient for publication of this case report. The patient refused image publication.

\section{References}

1. Rosenbaum E, Krebs E, Cohen M, Tiliakos A, Derk CT. The spectrum of clinical manifestations, outcome and treatment of pericardial tamponade in patients with systemic lupus erythematosus: a retrospective study and literature review. Lupus. 2009;18(7):608-612.

2. Nossent J, Kiss E, Rozman B, Pokorny G, Vlachoyiannopoulos P, Olesinska M, Marchesoni A, et al. Disease activity and damage accrual during the early disease course in a multinational inception cohort of patients with systemic lupus erythematosus. Lupus. 2010;19(8):949-956.

3. Shah MA, Shah AM, Krishnan E. Poor outcomes after acute myocardial infarction in systemic lupus erythematosus. J Rheumatol. 2009;36(3):570-575. 
4. Ansari A, Larson PH. Heart disease in systemic lupus erythematosus: diagnosis and management. Tex Heart Inst J. 1985;12(1):9-21.

5. Manzi S, Meilahn EN, Rairie JE, Conte CG, Medsger TA, Jr., Jansen-McWilliams L, D'Agostino RB, et al. Agespecific incidence rates of myocardial infarction and angina in women with systemic lupus erythematosus: comparison with the Framingham Study. Am J Epidemiol. 1997;145(5):408-415.

6. Livingston B, Bonner A, Pope J. Differences in clinical manifestations between childhood-onset lupus and adultonset lupus: a meta-analysis. Lupus. 2011;20(13):13451355 .

7. Edworthy SM. Clinical Manifestations of Systemic Lupus Erythematosus. In: Harris ED, et al, eds. Kelley's Textbook of Rheumatology. 7th ed. Philadelphia, Pa: WB Saunders; 2005. p. 1201-1224.

8. Shiel WC, Jr. Systemic Lupus Erythematosus (SLE or Lupus), Medicine net; 2015; retrieved from http://www. medicinenet.com/systemic_lupus/page4.htm.

9. Manson JJ, Rahman A. Systemic lupus erythematosus. Orphanet J Rare Dis. 2006;1:6.
10. Amissah-Arthur MB, Gordon C. Contemporary treatment of systemic lupus erythematosus: an update for clinicians. Ther Adv Chronic Dis. 2010;1(4):163-175.

11. E Borham L. Pharmacotherapy in Systemic Lupus Erythematosus. Current Rheumatology Reviews. 2012;8(3):166-179.

12. Hahn BH. Management of Systemic Lupus Erythematosus. In: Harris ED, et al, eds. Kelley's Textbook of Rheumatology. 7th ed. Philadelphia, Pa: WB Saunders; 2005. p. $1225-1247$.

13. Bertsias G, Ioannidis JP, Boletis J, Bombardieri S, Cervera $\mathrm{R}$, Dostal C, Font J, et al. EULAR recommendations for the management of systemic lupus erythematosus. Report of a Task Force of the EULAR Standing Committee for International Clinical Studies Including Therapeutics. Ann Rheum Dis. 2008;67(2):195-205.

14. Mosca M, Tani C, Aringer M, Bombardieri S, Boumpas D, Brey R, Cervera R, et al. European League Against Rheumatism recommendations for monitoring patients with systemic lupus erythematosus in clinical practice and in observational studies. Ann Rheum Dis. 2010;69(7):12691274. 